PHYSICAL REVIEW D 93, 129904(E) (2016)

\title{
Erratum: Asymptotic safety in an interacting system of gravity and scalar matter [Phys. Rev. D 93, 044049 (2016)]
}

Pietro Donà, Astrid Eichhorn, Peter Labus, and Roberto Percacci

(Received 15 May 2016; published 17 June 2016)

DOI: $10.1103 /$ PhysRevD.93.129904

In the following all equations have the same number as in the paper. There is a typo in the result for the anomalous dimension of the transverse traceless mode, which should read

$$
\begin{aligned}
\eta_{\mathrm{TT}}= & N_{S} \frac{1}{24 \pi} g_{3}+\frac{1}{1728 \pi} G_{3}\left(-2928+455 \eta_{\mathrm{TT}}-35 \eta_{\sigma}\right) \\
& -\frac{29}{648 \pi} G_{4}\left(-18+5 \eta_{\mathrm{TT}}-2 \eta_{\sigma}\right) .
\end{aligned}
$$

There was also an error in the derivation of the beta function of $g_{3}$, which did not correspond to a symmetric external momentum configuration, as stated. For the symmetric momentum configuration, we obtain the following results leading to $\beta_{g_{3}}$. The three-vertex-diagrams yield

$$
\begin{aligned}
& \left.\beta_{\sqrt{g_{3}}}\right|_{\mathrm{TT} 3 \text {-vertex }}=0, \\
& \left.\beta_{\sqrt{g_{3}}}\right|_{\sigma 3 \text {-vertex }}=\frac{1}{80 \pi} g_{3}^{3 / 2}\left(30-\eta_{\sigma}-2 \eta_{S}\right) .
\end{aligned}
$$

From the tadpoles, we obtain

$$
\begin{gathered}
\left.\beta_{\sqrt{g_{3}}}\right|_{\mathrm{TT} \text { tadpole }}=-\frac{95}{648 \pi} g_{5}^{3 / 2}\left(6-\eta_{\mathrm{TT}}\right), \\
\left.\beta_{\sqrt{g_{3}}}\right|_{\sigma \text { tadpole }}=\frac{19}{324 \pi} g_{5}^{3 / 2}\left(6-\eta_{\sigma}\right) .
\end{gathered}
$$

The two-vertex diagrams with only gravity-matter vertices are given by

$$
\begin{aligned}
& \left.\beta_{\sqrt{g_{3}}}\right|_{\text {two-vertex }} ^{\text {TTS }}=0, \\
& \beta_{\left.\sqrt{g_{3}}\right|_{\text {two-vertex }}}=-\frac{5}{72 \pi} \sqrt{g_{3}} g_{4}\left(16-\eta_{\sigma}-\eta_{S}\right) .
\end{aligned}
$$

Finally, the two- and three-vertex diagrams which also contain pure-graviton vertices give

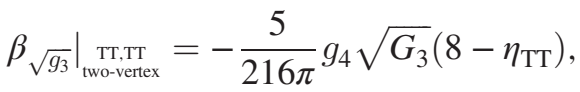

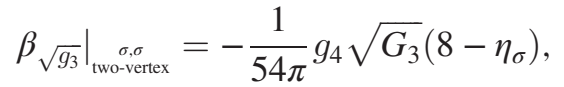

$$
\begin{aligned}
& \left.\beta_{\sqrt{g_{3}}}\right|_{\substack{\text { two-vertex } \\
\text { GT }}}=0 \text {, }
\end{aligned}
$$

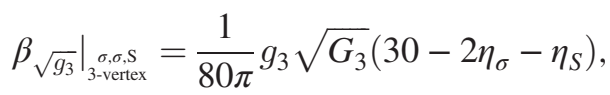

$$
\begin{aligned}
& \left.\beta_{\sqrt{g_{3}}}\right|_{\substack{\mathrm{TTT} \text {-Tr, } \\
\text {-vertex }}}=0,
\end{aligned}
$$


TABLE I. Coordinates and critical exponents at an interacting fixed point for vanishing scalar fluctuations.

\begin{tabular}{lcccc}
\hline \hline approximation & $g_{3 *}$ & $\theta$ & $\eta_{\mathrm{TT}}$ & $\eta_{\sigma}$ \\
\hline full & 2.204 & 2.17 & -0.62 & 0.50 \\
semi-pert. & 2.203 & 2.17 & -0.62 & 0.37 \\
pert. $\left(\eta_{\mathrm{TT}}=0=\eta_{\sigma}\right)$ & 3.65 & 2 & - & - \\
\hline \hline
\end{tabular}

TABLE II. Coordinates and critical exponents at an interacting fixed point at selected values of $N_{S}$. In this approximation, the fixed point disappears into the complex plane at $N_{S}=28$.

\begin{tabular}{lcccc}
\hline \hline$N_{S}$ & $g_{3 *}$ & $\theta$ & $\eta_{\mathrm{TT}}$ & $\eta_{\sigma}$ \\
\hline 1 & 1.82 & 2.09 & -0.48 & 0.49 \\
2 & 1.85 & 2.08 & -0.46 & 0.59 \\
5 & 1.95 & 2.05 & -0.39 & 0.91 \\
10 & 2.14 & 1.99 & -0.26 & 1.54 \\
15 & 2.40 & 1.90 & -0.08 & 2.31. \\
\hline \hline
\end{tabular}

$$
\beta_{\left.\sqrt{g_{3}}\right|_{\text {3-vertex }}}=0
$$

By summing Eqs. (41) and (51), we obtain the beta function for $\sqrt{g_{3}}$, from which we derive the beta function for the dimensionless $g_{3}$ as
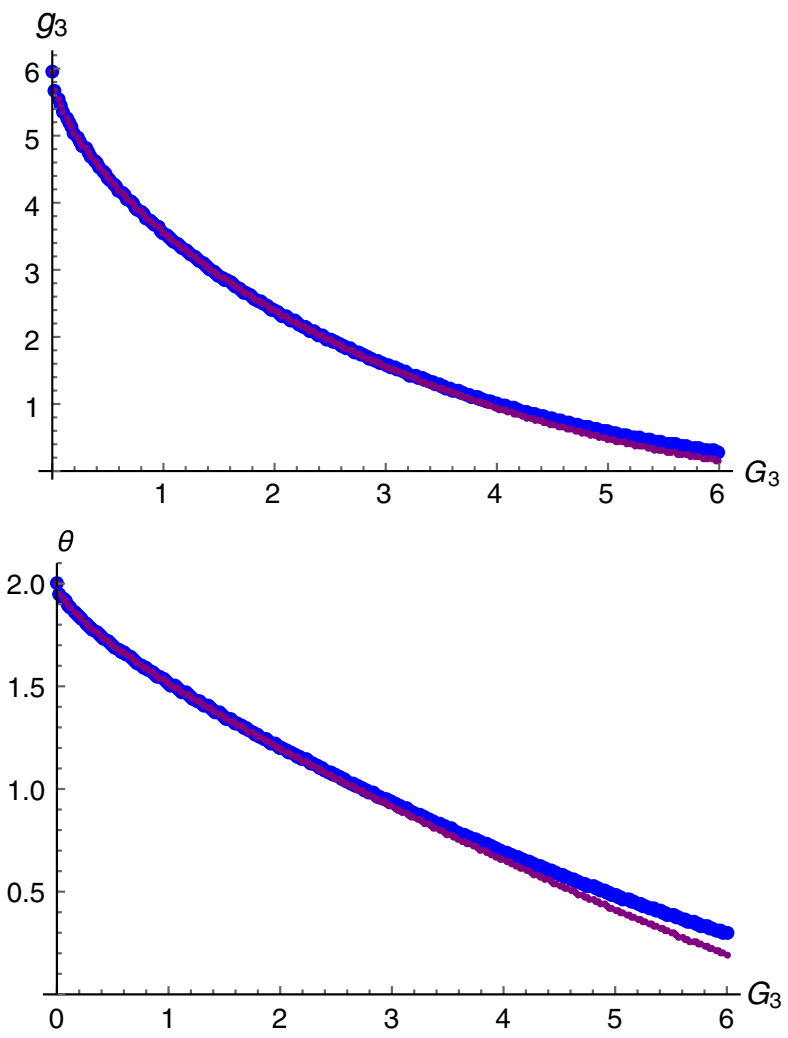

FIG. 8. We show the fixed-point value for $g_{3}$ (upper panel) and the critical exponent $\theta$ (lower panel) as a function of $G_{3}=G_{4}$. The larger blue dots denote the full result and the smaller purple dots denote the semiperturbative approximation. 

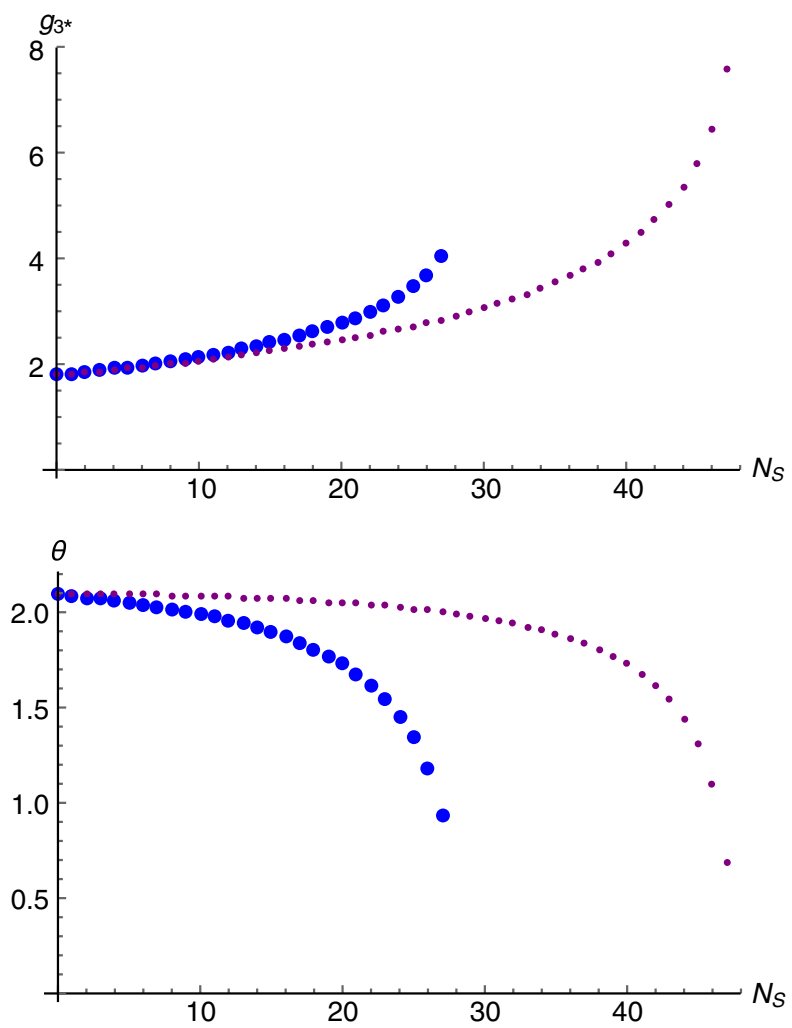

FIG. 10. We show the fixed-point value for $g_{3}$ (upper panel) and the critical exponent $\theta$ (lower panel) as a function of $N_{S}$. The larger blue dots denote the full result and the smaller purple dots denote the semiperturbative approximation.

$$
\begin{aligned}
\beta_{g_{3}}= & \left(2+\eta_{\mathrm{TT}}+2 \eta_{S}\right) g_{3}+\frac{3}{4 \pi} g_{3}^{2}+\frac{3}{4 \pi} g_{3}^{3 / 2} \sqrt{G_{3}} \\
& -\frac{20}{9 \pi} g_{3} g_{4}-\frac{2}{3 \pi} \sqrt{g_{3}} \sqrt{G_{3}} g_{4}-\frac{19}{18 \pi} g_{5}^{3 / 2} \sqrt{g_{3}} \\
& +\left(\frac{5}{108 \pi} g_{4} \sqrt{G_{3}}+\frac{95}{324 \pi} g_{5}^{3 / 2}\right) \sqrt{g_{3}} \eta_{\mathrm{TT}} \\
& +\left(-\frac{1}{40 \pi} g_{3}^{3 / 2}-\frac{1}{20 \pi} g_{3} \sqrt{G_{3}}+\frac{5}{36 \pi} \sqrt{g_{3}} g_{4}\right. \\
& \left.+\frac{1}{27 \pi} g_{4} \sqrt{G_{3}}-\frac{19}{162 \pi} g_{5}^{3 / 2}\right) \sqrt{g_{3}} \eta_{\sigma} \\
& +\left(-\frac{1}{20 \pi} g_{3}^{3 / 2}-\frac{1}{40 \pi} g_{3} \sqrt{G_{3}}+\frac{5}{36 \pi} \sqrt{g_{3}} g_{4}\right) \sqrt{g_{3}} \eta_{S} .
\end{aligned}
$$
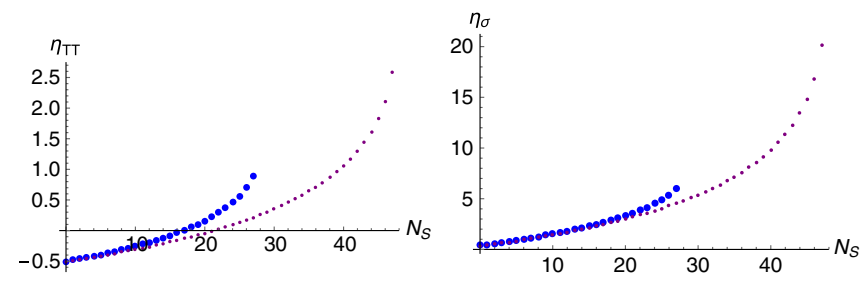

FIG. 11. We show the fixed-point value for $\eta_{\mathrm{TT}}$ (left panel) and $\eta_{\sigma}$ (right panel) as a function of $N_{S}$. The larger blue dots denote the full result and the smaller purple dots denote the semi perturbative approximation. 

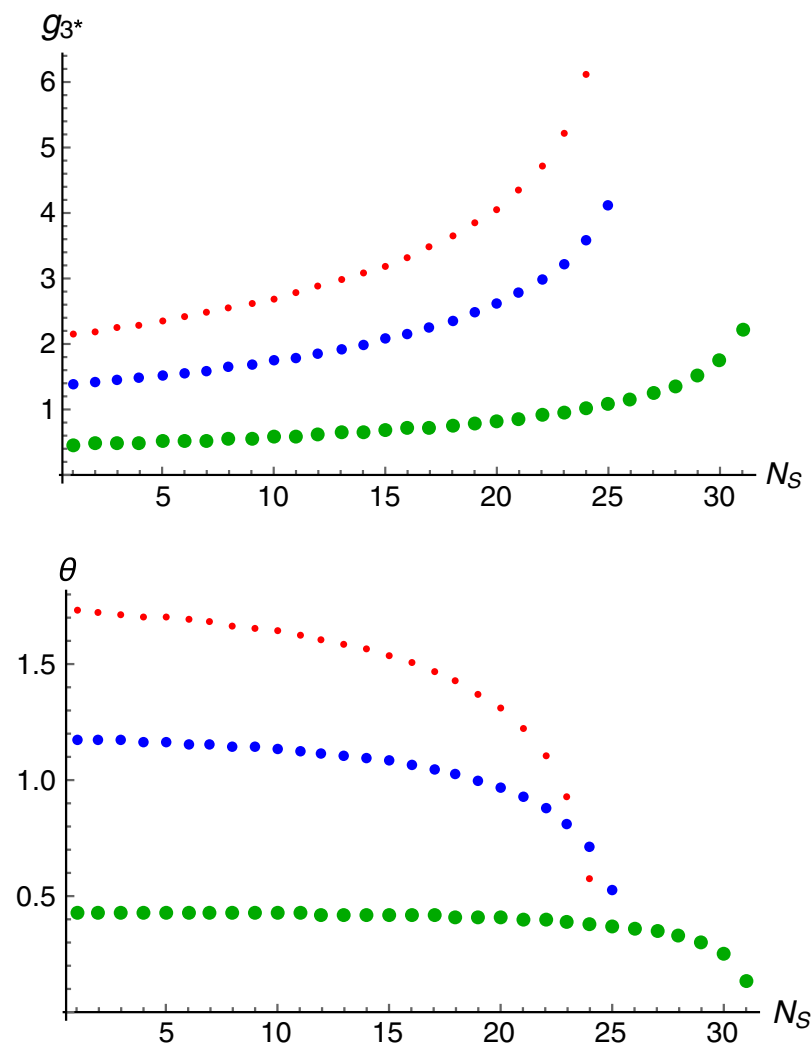

FIG. 12. We show the fixed-point value for $g_{3}$ (upper panel) and the critical exponent $\theta$ (lower panel) as a function of $N_{S}$. The small red dots are for $G_{3}=G_{4}=1$, the medium blue ones are for $G_{3}=G_{4}=3$ and the large green ones are for $G_{3}=G_{4}=6$.
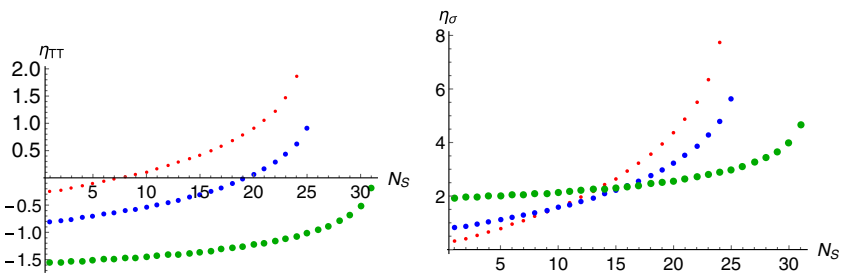

FIG. 13. We show the fixed-point value for $\eta_{\mathrm{TT}}$ (left panel) and for $\eta_{\sigma}$ (right panel) as a function of $N_{S}$. The small red dots are for $G_{3}=G_{4}=1$, the medium blue ones are for $G_{3}=G_{4}=3$ and the large green ones are for $G_{3}=G_{4}=6$. 


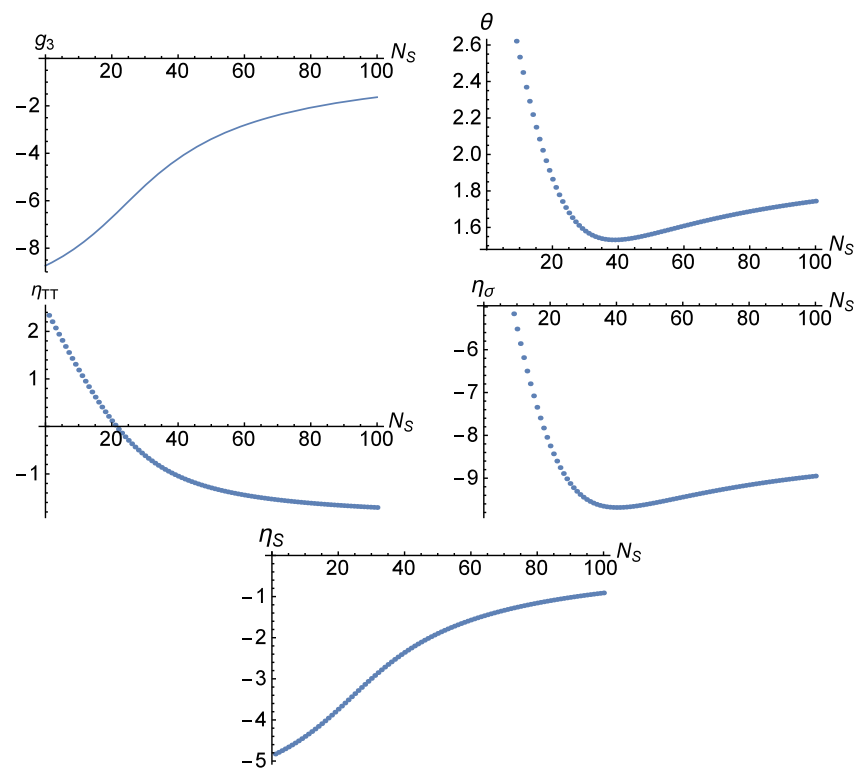

FIG. 14. We show results in the semiperturbative approximation: We plot the fixed-point value for $g_{3}$ as a function of $N_{S}$ (left upper panel) and the value of the critical exponent $\theta$ (right upper panel), as well as the anomalous dimensions.

The correct beta function leads to slightly different results for the fixed points. In the pure-gravity case, where all internal scalar lines are set to zero, and where we also set $\eta_{S}=0$, cf. Table I.

Including scalar fluctuations but keeping $\eta_{S}=0$, we obtain the fixed-point results in Table II in the approximation $G_{4}=G_{3}=g_{5}=g_{4}=g_{3}$.

The changes in the beta function reflect themselves in the figures, except for Fig. 9 which is not affected. We report below all the corrected figures. 